\title{
A simple and high-throughput method for xylem sap collection
}

Manman Kan ${ }^{\ddagger 1}$, Kiyoshi Yamazaki ${ }^{\ddagger, 1}$, Toru Fujiwara' \& Takehiro Kamiya ${ }^{\star, 1,2}$

\section{ABSTRACT}

Xylem is the most important route for root-to-shoot translocation of water, nutrients and signaling molecules. Although a number of studies have been performed using xylem sap, its collection requires special equipment and is usually low throughput. Here, we developed a simple and highthroughput method for the collection of both medium- $(<1 \mathrm{ml})$ and small$(<200 \mu \mathrm{l})$ volume xylem sap samples. Using a rice $\mathrm{Cd}$ transporter mutant, we demonstrated that our method allows for the effective evaluation of xylem sap Cd concentrations.

\section{METHOD SUMMARY}

Here, we describe a simple and highthroughput method for the collection of medium $(<1 \mathrm{ml})$ and small $(<200 \mu \mathrm{l})$ xylem sap samples. Xylem sap is collected in 1.5-, 2- or 5-ml plastic tubes containing cotton, without the use of special instruments. The xylem sap collected using this method can be used directly for ionome analysis.

KEYWORDS

ionome $\cdot$ translocation $\cdot$ xylem $\cdot$ xylem sap

'Department of Applied Biological Chemistry, Graduate School of Agricultural \& Life Sciences, The University of Tokyo, 1-7-1, Yayoi, Bunkyo, Tokyo, 113-8657, Japan; ${ }^{2}$ Precursory Research for Embryonic Science \& Technology (PRESTO), Japan Science \& Technology Agency (JST), 4-1-8 Honcho Kawaguchi, Saitama, 332-0012, Japan; *Author for correspondence: akamiyat@mail.

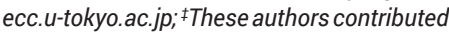
equally to this work

BioTechniques 67: 242-245 (November 2019) $10.2144 /$ btn-2019-0046
Xylem plays an important role in water and nutrient transport from roots to shoots in land plants [1]. The long-distance transport of elements and signaling peptides through xylem sap has recently gained attention from researchers [2-5] and xylem sap collection methods have been developed accordingly [6]. Xylem sap is a term usually used for an exudate from the cut shoot end [6], and therefore the sap contains a portion of phloem sap and sap from damaged cells. To date, several methods for xylem sap collection have been employed. Some of these, such as the pressure vessel equipment method $[7,8]$ and the vacuum pump method [9], require special equipment and techniques. Methods based on root pressure using microcapillary tubes, micropipettes or plastic tubes [2,10] do not require special instruments, but require experience and continuous attention during sap collection. Although these methods have been employed in a number of studies, they are low throughput. Here, we established a simple and high-throughput xylem sap collection method that requires only tubes and cotton, without the use of special techniques or the need to monitor xylem sap exudation during the sap collection process.

Our method can be used to collect small $(<200 \mu \mathrm{l})$ or medium ( $<1 \mathrm{ml}$ ) samples using the following general laboratory equipment: 1.5-, 2- and 5-ml plastic tubes, absorbent cotton and small pieces of plastic straws. The collection process for small samples is illustrated in Figure 1A \& F. First, absorbent cotton is inserted into plastic strawcontaining 1.5-ml tubes (131-715C, Watson; small samples) or 2-ml tubes (132-620C, Watson; medium samples; Figure 1B \& G). These cotton-containing tubes are then placed on the cut ends of shoots (Figure 1C \& $\mathrm{H})$ and xylem sap flowing from the cut shoot is absorbed and collected in the cotton inside the tubes. We recommend using ceramic scissors to cut shoots to prevent the risk of metal contamination when sap is used for element determination. The tubes are left on the cut shoot ends for up to $24 \mathrm{~h}$. During collection, the sap evaporates, and therefore a short duration would be recommended to avoid the loss of xylem sap through evaporation. In median scale, with $120 \mu$ of water, $82 \pm 2 \mu \mathrm{l}(\mathrm{n}=3$, mean $\pm \mathrm{SD})$ of water was recovered after $24-h$ incubation under the same condition as rice growth, indicating that the variation of evaporation was small and used for relative comparison. After collection, xylem sap can be stored at $4^{\circ} \mathrm{C}$ or $-20^{\circ} \mathrm{C}$ until further processing.

To recover the xylem sap absorbed in the cotton, 1.5-ml tubes can be subjected to centrifugation directly at $15,000 \times g$ for $2 \mathrm{~min}$, thereby separating the xylem sap from the cotton kept above the plastic straw and collected in the bottom of the 1.5-ml tubes. Using this method, up to $200-\mu$ xylem sap can be recovered. This small-scale method is suitable for use in young rice or sorghum seedlings with small shoots, as the collection tubes are lightweight. This method is also easily and widely applicable as it requires 1.5-ml tubes, which are generally found in all laboratories. For medium-volume sample collection, a hole is made at the bottom of the tubes using a pin, and the tubes are placed into 5-ml tubes (ST-500, BIO-BIK; Figure 1J). The 5-ml tubes set with 2-ml tubes are placed into $15-\mathrm{ml}$ centrifuge tubes (339650, Thermo Fisher Scientific; adapted for centrifugation) and centrifuged at $15,000 \times g$ for $3 \mathrm{~min}$ to separate the collected xylem sap from the cotton in the 2-ml tubes, which is squeezed into the bottom of the $5-\mathrm{ml}$ tube. Using this method, up to $1 \mathrm{ml}$ xylem sap can be collected.

Using the method developed here, we successfully collected xylem sap from rice and sorghum plants. In the case of sorghum, plants were grown in 50-ml tubes containing 45- $\mathrm{ml}$ quarter-strength Hoagland's nutrient solution [11] in a growth chamber at $30^{\circ} \mathrm{C}$ with $16 \mathrm{~h}$ light and $8 \mathrm{~h}$ dark cycles (Figure $1 \mathrm{C}$ ). The plants were held upright with rockwool. After cultivation for 2 weeks, the seedling shoots were cut $5 \mathrm{~cm}$ above the rockwool using 

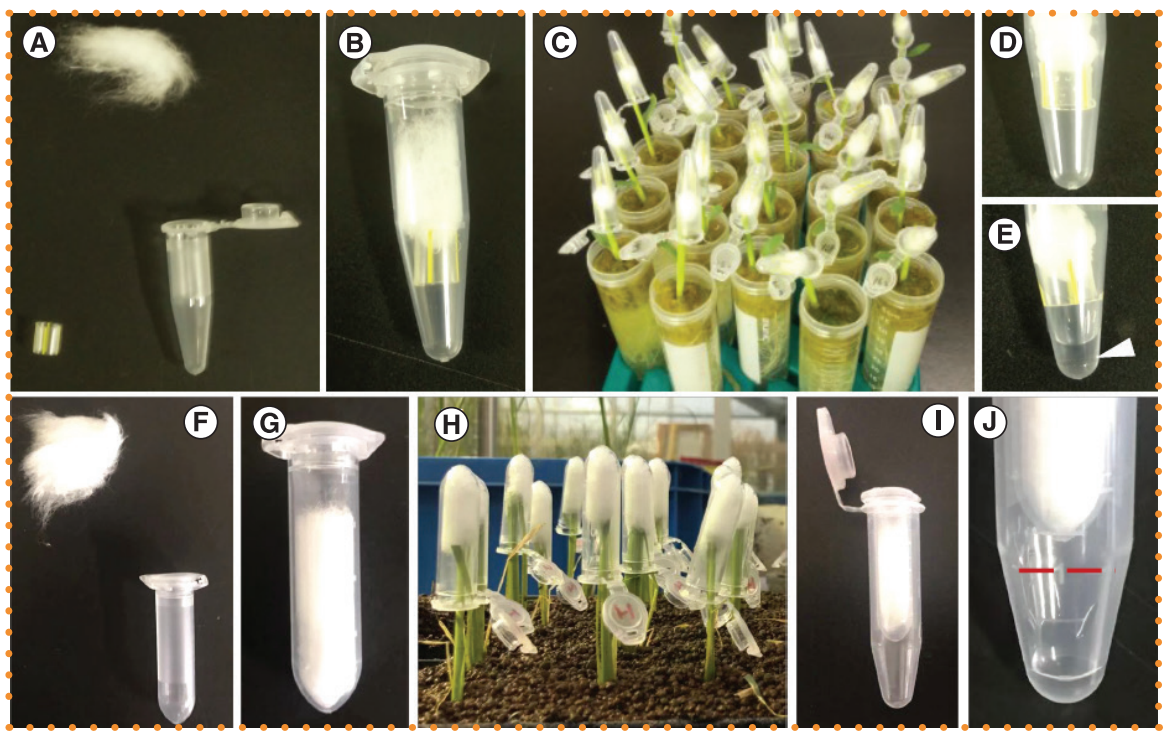

Figure 1. Xylem sap collection of small- and medium-volume samples using cotton and tubes. (A-D) Xylem sap collection of small-volume samples. (A) Items required for small sample collection: plastic straws, absorbent cotton and $1.5-\mathrm{ml}$ tubes. (B) Tube assembly for xylem sap collection. (C) Assembled tubes are placed on cut shoots and the xylem sap is absorbed by the cotton. (D) After xylem sap absorption in cotton, tubes are centrifuged $(15,000 \times \mathrm{g}$ for $2 \mathrm{~min})$. (E) The absorbed xylem sap is separated from the cotton and collected in the bottom of the tubes (arrowhead). ( $F-J)$ Xylem sap collection of medium-volume samples. ( $F$ ) Items required for medium sample collection: absorbent cotton and 2-ml tubes. (G) Assembly for xylem sap collection. (H) Assembled tubes are placed on cut shoots and the xylem sap is absorbed by the cotton. (I) After xylem sap absorption, a hole is made at the bottom of the tube using a pin and the tube is placed in a 5-ml tube. $(\mathrm{J})$ The absorbed xylem sap is separated from the cotton and collected in the bottom of the 5 - $\mathrm{ml}$ tube via centrifugation $(15,000 \times \mathrm{g}$ for $3 \mathrm{~min}$ ). The red dashed line represents the maximum volume that can be collected using this medium sample collection method $(1 \mathrm{ml})$.
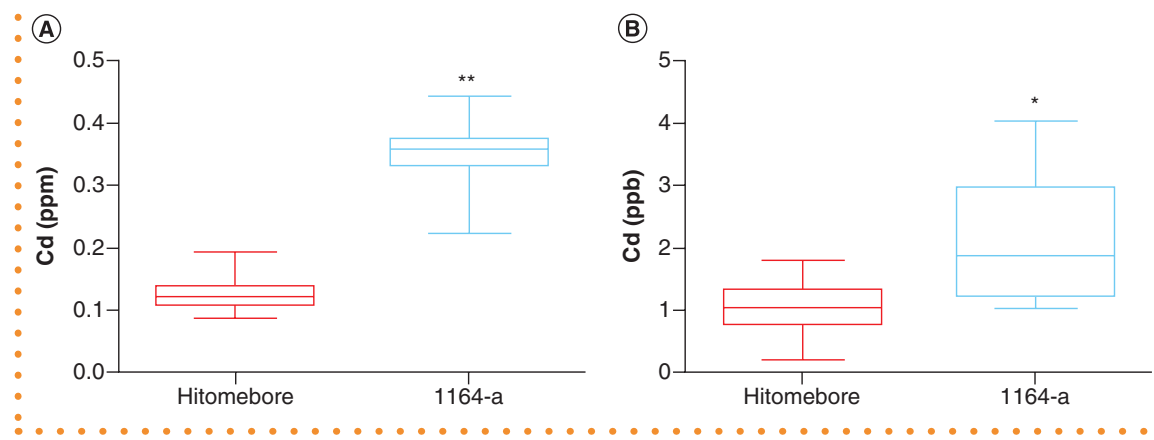

Figure 2. Cd concentrations in shoots and xylem sap. $C d$ concentrations in the shoots $(A)$ and xylem sap collected using the medium sample collection method (B). 5-week-old plants were used for the analysis. 1164-a is an OsHMA3 mutant and Hitomebore is the corresponding wild-type. (A) $n=14 ;(B) n=9-14$. Mann-Whitney-Wilcoxon test. ${ }^{*} \mathrm{p}<0.05 ; * \star \mathrm{p}<0.01$.

- scissors, and $1.5 \mathrm{ml}$ tubes containing cotton were placed on the cut end of the shoots for 10-15 s per plant (small-volume method). Approximately $50 \mu \mathrm{l}$ xylem sap was collected after $24 \mathrm{~h}$ (Figure 1E). In the case of rice, plants were cultivated in commercial soil for 5 weeks in a growth chamber under the same conditions as those used for sorghum. Seedlings were cut $5 \mathrm{~cm}$ above the soil and 2-ml tubes containing cotton were placed on the cut shoots. After $24 \mathrm{~h}$, approximately $120 \mu \mathrm{l}$ xylem sap was collected (Figure 1J).

To confirm whether the collected sap could be used to evaluate xylem sap properties, $\mathrm{Cd}$ concentrations in the xylem sap collected using the medium-volume collection method were evaluated, and those of a OsHMA3 rice mutant (1164-a) [12] were compared with those of the wild-type
(Hitomebore). OsHMA3 is a P1B-type ATPase that regulates root-to-shoot $\mathrm{Cd}$ translocation and mutants show high $\mathrm{Cd}$ accumulation in shoots [3,13]. The shoots of 5-week-old rice plants grown in commercial soil were cut $5 \mathrm{~cm}$ above the soil using scissors and shoots were harvested together with xylem sap to measure $\mathrm{Cd}$ concentrations. The dry weights of the shoots were measured and digested with $\mathrm{HNO}_{3}$. After dissolving digested samples in $0.08 \mathrm{M} \mathrm{HNO}_{3}$ containing $2 \mathrm{ppb}$ In (internal control), Cd concentrations were determined via inductively coupled plasma mass spectrometry (ICP-MS; Agilent 7800). As expected, Cd concentrations in the mutant shoots were significantly higher than those in the wild-type ( $n=18, p<0.01$; Figure 2A). To measure Cd concentrations, xylem sap was diluted with $0.08 \mathrm{M} \mathrm{HNO}_{3}$ containing $2 \mathrm{ppb}$ In, and was directly analyzed via ICP-MS. Cd concentrations in the xylem sap of the mutants were higher than those in the wild-type $(n=9-14, p<0.05)$, indicating the validity of our method.

We measured concentrations of 23 elements including $\mathrm{Cd}$ in the xylem sap and also measured element contamination from the assembly (cotton and/or straw and tubes; Table 1). To determine the amount of contamination (see Table 1), instead of xylem sap, a similar amount of water was applied to the assembly followed by centrifugation. We were able to quantify the concentrations of 19 elements in the xylem sap of both rice and sorghum plants. Among them, most of the elements showed higher concentrations than the contamination from the assembly. These results indicate that our method is appropriate for ionomic analysis with attention to the contamination.

In this paper, to establish high-throughput analysis we removed a step that prevents phloem sap contamination [6]. In addition, in order to process a lot of samples, we collected xylem sap long term (24 h). During $24 \mathrm{~h}$, the plants would be damaged and the sap would evaporate, which led to the inaccurate measurement of absolute element concentrations. Therefore, to obtain accurate concentrations, another method should be performed after the measurement by our method, as described by Alexou and Peuke [6] and our method with modifications: removal of the very first drops of xylem 


\begin{tabular}{|c|c|c|c|c|c|}
\hline \multirow[t]{3}{*}{ Element } & \multicolumn{3}{|c|}{ Medium-scale method } & \multicolumn{2}{|c|}{ Small-scale method } \\
\hline & \multirow{2}{*}{$\begin{array}{l}\text { Contamination from } \\
\text { the assembly }(n=3)\end{array}$} & \multicolumn{2}{|c|}{ Xylem sap (rice) } & \multirow{2}{*}{$\begin{array}{l}\text { Contamination from } \\
\text { the assembly }(n=4)\end{array}$} & \multirow{2}{*}{$\begin{array}{l}\text { Xylem sap (sorghum) } \\
(\mathrm{n}=110)\end{array}$} \\
\hline & & $\begin{array}{l}\text { Hitomebore } \\
(n=14)\end{array}$ & $1164-a(n=9)$ & & \\
\hline $\mathrm{Li}$ & $1.21 \pm 0.00$ & $2.19 \pm 1.10$ & $2.91 \pm 2.29$ & $1.98 \pm 0.29$ & $9.51 \pm 3.98$ \\
\hline B & $138 \pm 37$ & $386 \pm 76$ & $405 \pm 53$ & $316 \pm 350$ & $892 \pm 369$ \\
\hline $\mathrm{Na}$ & $5070 \pm 130$ & $13,200 \pm 8500$ & $18,000 \pm 4300$ & $6300 \pm 920$ & $10,700 \pm 6700$ \\
\hline $\mathrm{Mg}$ & $1300 \pm 90$ & $24,200 \pm 6800$ & $29,300 \pm 6800$ & $1170 \pm 300$ & $41,100 \pm 20,900$ \\
\hline$P$ & $962 \pm 34$ & ND & ND & $667 \pm 82$ & $78,300 \pm 51,300$ \\
\hline$s$ & ND & ND & ND & ND & $24,300 \pm 21,000$ \\
\hline K & 3460 & $308,000 \pm 152,000$ & $374,000 \pm 139,000$ & $5520 \pm 2020$ & $614,000 \pm 31,7000$ \\
\hline $\mathrm{Ca}$ & $4810 \pm 240$ & $77,400 \pm 26,500$ & $83,500 \pm 19,400$ & $4970 \pm 1030$ & $94,000 \pm 45,800$ \\
\hline Mn & $17.6 \pm 5.1$ & $31,600 \pm 15,300$ & $28,300 \pm 13,600$ & $12.1 \pm 1.7$ & $2660 \pm 1400$ \\
\hline $\mathrm{Fe}$ & ND & $65.8 \pm 43.8$ & $172 \pm 111$ & ND & $589 \pm 825$ \\
\hline Co & ND & $3.93 \pm 2.01$ & $6.78 \pm 1.63$ & ND & $10.7 \pm 18.6$ \\
\hline $\mathrm{Ni}$ & ND & $10.7 \pm 6.3$ & $17.3 \pm 17.1$ & ND & $13.6 \pm 16.1$ \\
\hline $\mathrm{Cu}$ & 10.4 & $66.4 \pm 29.6$ & $104 \pm 48$ & ND & $324 \pm 238$ \\
\hline $\mathrm{Zn}$ & ND & $1110 \pm 393$ & $1650 \pm 1080$ & ND & $786 \pm 587$ \\
\hline $\mathrm{Ge}$ & ND & $1.66 \pm 0.53$ & $1.84 \pm 0.53$ & ND & $0.274 \pm 0.091$ \\
\hline As & $0.29 \pm 0.08$ & $0.317 \pm 0.125$ & $0.483 \pm 0.243$ & $0.307 \pm 0.037$ & $0.412 \pm 0.197$ \\
\hline Se & ND & ND & ND & ND & ND \\
\hline $\mathrm{Rb}$ & 1.98 & $456 \pm 210$ & $558 \pm 200$ & $2.32 \pm 1.87$ & $131 \pm 65$ \\
\hline $\mathrm{Sr}$ & $9.7 \pm 1.0$ & $93.8 \pm 34.9$ & $115 \pm 22$ & $10.1 \pm 1.7$ & $135 \pm 70$ \\
\hline Mo & $0.839 \pm 0.207$ & $3.79 \pm 3.42$ & $3.59 \pm 1.75$ & $1.48 \pm 0.91$ & $80.7 \pm 43.6$ \\
\hline $\mathrm{Cd}$ & ND & $1.03 \pm 0.48$ & $2.09 \pm 1.04$ & ND & $0.271 \pm 0.159$ \\
\hline Cs & $0.124 \pm 0.035$ & $3.05 \pm 1.02$ & $3.73 \pm 1.06$ & $0.0943 \pm 0.0149$ & $1.07 \pm 0.49$ \\
\hline
\end{tabular}

sap [6] and a short-term harvest time, such as $1-3 \mathrm{~h}$.

The xylem sap collection methods developed in this study are simple, user friendly, high throughput and cost effective, and will be helpful for studies on xylem sap. These methods could also be applicable for other omics analyses, such as metabolomic and proteomic studies, as well as ionomic studies.

\section{ACKNOWLEDGMENTS}

The authors would like to thank Emiko Yokota for technical assistance.

\section{AUTHOR CONTRIBUTIONS}

$\mathrm{KM}$ and $\mathrm{KY}$ contributed equally. $\mathrm{KM}, \mathrm{KY}$ and TK designed and performed the experiments. $\mathrm{KM}$ analyzed data. $\mathrm{KM}, \mathrm{KY}$ and TK prepared the manuscript. TF and TK supervised the study and corrected the manuscript.

\section{FINANCIAL \& COMPETING INTERESTS DISCLOSURE}

This work was supported by JST, PRESTO grant number JPMJPR16Q3 to TK, Japan. Grant-in-Aid for scientific research to TF (no. 25221202). The authors have no other relevant affiliations or financial involvement with any organization or entity with a financial interest in or financial conflict with the subject matter or materials discussed in the manuscript apart from those disclosed.

No writing assistance was utilized in the production of this manuscript.

\section{OPEN ACCESS}

This work is licensed under the AttributionNonCommercial-NoDerivatives 4.0 Unported License. To view a copy of this license, visit http://creativecommons.org/licenses/ by-nc-nd/4.0/ 


\section{REFERENCES}

1. Rodríguez-Celma J, Ceballos-Laita L, Grusak MA, Abadía J, López-Millán AF. Plant fluid proteomics: delving into the xylem sap, phloem sap and apoplastic fluid proteomes. Biochim. Biophys. Acta. 1864(8), 991-1002 (2016).

2. Mitani N, Ma JF, Iwashita T. Identification of the silicon form in xylem sap of rice (Oryza sativa L.). Plant Cell Physiol. 46(2), 279-283 (2005).

3. Miyadate $\mathrm{H}$, Adachi $\mathrm{S}$, Hiraizumi $\mathrm{A}$ et al. OsHMA3, a P-1B-type of ATPase affects root-to-shoot cadmium translocation in rice by mediating efflux into vacuoles. New Phytol. 189(1), 190-199 (2011)

4. González JF, Degrassi G, Devescovi G et al. A proteomic sap

5. Li W, Jia L, Wang L. Chemical signals and their regulations on the plant growth and water use efficiency of cotton seedlings under partial root-zone drying and different nitrogen applications. Saudi. J. Biol. Sci. 24(3), 477-487 (2017).

6. Alexou M, Peuke AD. Methods for xylem sap collection. Methods Mol. Biol. 953, 195-207 (2013).

7. Scholander PF, Bradstreet ED, Hemmingsen EA, Hammel HT. Sap pressure in vascular plants: negative hydrostatic pressure can be measured in plants. Science 148(3668), 339-346 (1965).

8. Passioura JB. The transport of water from soil to shoot in wheat seedlings. J. Exp. Bot. 31(120), 333-345 (1980).

9. Milburn JA, Ranasinghe MS. A comparison of methods for studying pressure and solute potentials in xylem and also in phloem laticifers of Hevea brasiliensis. also in phloem laticifers of Hevea brasilien
J. Exp. Bot. 294), 135-143 (1996).

10. Ariga T, Hazama $K$, Yanagisawa S, Yoneyama T. Chem ical forms of iron in xylem sap from graminaceous and non-graminaceous plants. Soil Sci. Plant Nut. 60(4), 460-469 (2014).

11. Hoagland DR, Arnon DI. The water-culture method for growing plants without soil. Calif. Agric. $347(2), 32$ (1950).

12. Tanaka N, Nishida S, Kamiya T, Fujiwara T. Large-scale profiling of brown rice ionome in an ethyl methanesulphonate-mutagenized hitomebore population and identification of high- and low-cadmium lines. Plant Soil 407(1-2), 109-117 (2016).

13. Ueno D, Yamaji N, Kono I et al. Gene limiting cadmium accumulation in rice. Proc. Natl Acad. Sci. USA 107(38), 16500-16505 (2010) 